\title{
Progression to microalbuminuria in type 1 diabetes: development and validation of a prediction rule
}

\author{
Y. Vergouwe • S. S. Soedamah-Muthu • J. Zgibor • N. Chaturvedi • C. Forsblom • \\ J. K. Snell-Bergeon • D. M. Maahs • P.-H. Groop • M. Rewers • T. J. Orchard • \\ J. H. Fuller • K. G. M. Moons
}

Received: 11 September 2009 / Accepted: 23 September 2009/Published online: 4 November 2009

(C) The Author(s) 2009. This article is published with open access at Springerlink.com

\begin{abstract}
Aims/hypothesis Microalbuminuria is common in type 1 diabetes and is associated with an increased risk of renal and cardiovascular disease. We aimed to develop and validate a clinical prediction rule that estimates the absolute risk of microalbuminuria.

Methods Data from the European Diabetes Prospective Complications Study $(n=1115)$ were used to develop the prediction rule (development set). Multivariable logistic regression analysis was used to assess the association between potential predictors and progression to microalbuminuria within 7 years. The performance of the prediction rule was
\end{abstract}

Electronic supplementary material The online version of this article (doi:10.1007/s00125-009-1585-3) contains a list of the members of the EURODIAB, EDC, FinnDiane and CACTI Study Groups and other contributors to the study, which is available to authorised users.

Y. Vergouwe $(\varangle) \cdot$ S. S. Soedamah-Muthu $\cdot$ K. G. M. Moons Julius Center for Health Sciences and Primary Care,

University Medical Centre Utrecht,

Str 6.131, P.O. Box 85500, 3508 GA Utrecht, the Netherlands

e-mail: y.vergouwe@umcutrecht.nl

S. S. Soedamah-Muthu

Division of Human Nutrition, Wageningen University,

Wageningen, the Netherlands

S. S. Soedamah-Muthu $\cdot$ J. H. Fuller

Department of Epidemiology and Public Health,

Royal Free and University College London Medical School,

London, UK

J. Zgibor · T. J. Orchard

Department of Epidemiology, Graduate School of Public Health,

University of Pittsburgh,

Pittsburgh, PA, USA assessed with calibration and discrimination (concordance statistic [c-statistic]) measures. The rule was validated in three other diabetes studies (Pittsburgh Epidemiology of Diabetes Complications [EDC] study, Finnish Diabetic Nephropathy [FinnDiane] study and Coronary Artery Calcification in Type 1 Diabetes [CACTI] study).

Results Of patients in the development set, $13 \%$ were microalbuminuric after 7 years. Glycosylated haemoglobin, AER, WHR, BMI and ever smoking were found to be the most important predictors. A high-risk group $(n=87$ [8\%]) was identified with a risk of progression to microalbuminuria of $32 \%$. Predictions showed reasonable discriminative
N. Chaturvedi

National Heart and Lung Institute,

Imperial College London,

London, UK

C. Forsblom • P.-H. Groop

Folkhälsan Institute of Genetics, Folkhälsan Research Center,

Biomedicum Helsinki,

Helsinki, Finland

C. Forsblom · P.-H. Groop

Division of Nephrology, Department of Medicine,

Helsinki University Central Hospital,

Helsinki, Finland

J. K. Snell-Bergeon · D. M. Maahs · M. Rewers

Barbara Davis Center for Childhood Diabetes,

University of Colorado,

Denver, Aurora, CO, USA 
ability, with $c$-statistic of 0.71 . The rule showed good calibration and discrimination in EDC, FinnDiane and CACTI (c-statistic $0.71,0.79$ and 0.79 , respectively).

Conclusions/interpretation We developed and validated a clinical prediction rule that uses relatively easily obtainable patient characteristics to predict microalbuminuria in patients with type 1 diabetes. This rule can help clinicians to decide on more frequent check-ups for patients at high risk of microalbuminuria in order to prevent long-term chronic complications.

Keywords Microalbuminuria risk · Prediction model · Progression - Type 1 diabetes

\begin{tabular}{|c|c|}
\hline \multicolumn{2}{|c|}{ Abbreviations } \\
\hline CACTI & $\begin{array}{l}\text { Coronary Artery Calcification in Type } 1 \\
\text { Diabetes }\end{array}$ \\
\hline c-statistic & Concordance statistic \\
\hline EDC & Epidemiology of Diabetes Complications \\
\hline EURODIAB & European Diabetes Prospective \\
\hline PCS & Complications Study \\
\hline FinnDiane & Finnish Diabetic Nephropathy study \\
\hline
\end{tabular}

\section{Introduction}

Microalbuminuria is common in type 1 diabetes. Of patients with type 1 diabetes, 30 to $60 \%$ progress to microalbuminuria within 10 to 20 years $[1,2]$. Various risk factors have been found for the progression to microalbuminuria including: hyperglycaemia, hyperlipidaemia, (central) obesity, hypertension and smoking [2-5].

Patients with microalbuminuria have elevated risks of diabetic renal disease [6,7] and cardiovascular disease [8]. Timely prediction of progression to microalbuminuria in patients with type 1 diabetes is therefore of major importance. Accurate identification of patients at high risk of microalbuminuria at an early stage can guide disease management in order to prevent further complications. Intervention in high-risk patients may be beneficial, although data to support this are inconclusive.

Prediction of microalbuminuria progression is rarely studied [2, 5]. Only one prediction rule has been proposed before [5]. This rule uses arbitrary dichotomisation of various continuous predictor values $\left(\mathrm{HbA}_{1 \mathrm{c}}\right.$ and $\left.\mathrm{AER}\right)$. Further, selection of the predictors was based on a $p$ value of 0.05 , which is considered conservative in prediction modelling. Both strategies potentially lead to an optimistic or overfitted prediction rule $[9,10]$. Moreover, the prediction rule was not validated in an independent sample.

Using state of the art methods in prediction modelling, we therefore developed and externally validated a clinical prediction rule for estimating risk of progression to microalbuminuria in patients with type 1 diabetes. We used data from large prospective cohort studies of type 1 diabetes. The rule was developed with data from the European Diabetes Prospective Complications Study (EURODIAB PCS) [11] and validated in US patients treated in a similar time period (Pittsburgh Epidemiology of Diabetes Complications [EDC]) study [1]. Since treatment policies have changed over the years, we also studied the validity of the rule in two more contemporary studies, i.e. the Finnish Diabetic Nephropathy study (FinnDiane) [12] and the Coronary Artery Calcification in Type 1 Diabetes study (CACTI) [13].

\section{Methods}

Patients We used data from the EURODIAB PCS to develop the prediction rule (development set). Full details of the design, methods and recruitment have been published elsewhere [11]. In brief, the study was designed to investigate risks and determinants of microvascular complications in type 1 diabetes patients. The EURODIAB PCS cohort included patients from 31 centres in 16 European countries.

Type 1 diabetes was defined as a diagnosis made before the age of 36 with continuous insulin treatment within 1 year of diagnosis. Patient measurements were taken at baseline (1990-1991) and at 7 years follow-up (19971999). Patients were included in the present analysis if they had a normal AER, i.e. below $20 \mu \mathrm{g} / \mathrm{min}$, at baseline and a normal AER or microalbuminuria (AER between 20 and $200 \mu \mathrm{g} / \mathrm{min}$ ) at follow-up.

We used data from three studies to externally validate the prediction rule (validation sets): the EDC study, FinnDiane and CACTI. The EDC study is a hospital-based incidence cohort from the Children's Hospital of Pittsburgh, USA, set up to investigate risk factors for complications of these patients [1]. After the baseline examination (1986-1988), patients were re-examined biennially. The FinnDiane study is a nationwide, multicentre study with the aim to identify genetic and clinical risk factors for diabetic nephropathy in type 1 diabetes [12]. Patients have been followed since the baseline examination (1998). The CACTI study is a prospective cohort study designed to assess risk factors associated with the development and progression of subclinical coronary artery disease [13]. Baseline examination was performed between 2000 and 2002. All three studies are ongoing. For the present analysis, follow-up measurements 7 years after inclusion were taken to assess the development of microalbuminuria. The same inclusion criteria were used as for the development set.

Outcome Microalbuminuria (yes/no) was determined 7 years after inclusion and defined as an AER between 20 
and $200 \mu \mathrm{g} / \mathrm{min}$. In EURODIAB PCS, the average of two $24 \mathrm{~h}$ urine samples was used for classification. Albumin concentrations were assessed with an immunoturbidimetric [14] method that included goat anti-human albumin antisera (Sanofi Diagnostics Pasteur, Chaska, MN, USA) and human serum albumin standards (ORHA 20/21 grade HAS; Behring Diagnostics, Hoechst UK, Hounslow, UK). Proteinuria due to urinary tract infection was excluded.

In the EDC study, an immuno-nephelometric method was used [15] and the median AER was calculated on the basis of three urine samples $(24 \mathrm{~h}$, overnight and $4 \mathrm{~h}$ postclinic) or the average AER if only two samples were available. In the FinnDiane study, AER was calculated from a single $24 \mathrm{~h}$ urine collection, which was measured by immunoturbidimetry. The actual classification of patients was based on two out of three consecutive urine samples. In the CACTI study, AER was calculated from urinary albumin measured using RIA in two timed overnight urine samples with the results from the two nights being averaged.

Candidate predictors The patient characteristics available in the EURODIAB PCS dataset were critically reviewed using the literature and clinical expertise. The following characteristics were considered to be possibly predictive for microalbuminuria: age, sex, duration of diabetes, $\mathrm{HbA}_{1 \mathrm{c}}$, AER, fasting triacylglycerol, non-HDL- and LDLcholesterol, WHR, BMI, pulse pressure, hypertension and smoking. $\mathrm{HbA}_{1 \mathrm{c}}$ was measured with an enzyme immunoassay using a monoclonal antibody. The $\mathrm{HbA}_{1 \mathrm{c}}$ values obtained were converted to DCCT values [16]. One $24 \mathrm{~h}$ urine sample was analysed to determine the AER. Serum lipids were measured with standard enzymatic methods. Non-HDL-cholesterol was calculated as the total cholesterol level minus the HDL level. Height, weight, and waist and hip circumference were measured in a standardised way to calculate BMI and WHR. Blood pressure was measured by a random zero sphygmomanometer and the mean of two measurements taken. Pulse pressure was calculated as systolic minus diastolic pressure. Hypertension was defined as a systolic pressure of $140 \mathrm{mmHg}$ or more, a diastolic pressure of $90 \mathrm{mmHg}$ or more, or the current use of blood-pressure-lowering medication. Smoking status was dichotomised into ever vs never smokers.

Statistical analysis In the EURODIAB PCS, 355 patients had values missing for one or more predictors. The average percentage of missing values per predictor was $3 \%$. We imputed missing data using the linear-regression method with addition of a random-error term (single imputation) available in SPSS software (version 12.0; SPSS, Chicago, IL, USA).

Logistic regression was used to estimate univariable and multivariable regression coefficients, and odds ratios with
95\% CIs for each predictor. The nature of the association between continuous predictors and risk of microalbuminuria was studied with restricted cubic splines with three knots (two $d f$ ). The restricted cubic splines were plotted and approximated with simple transformations. The simplest transformation is a linear term. Other possible transformations included the natural logarithm, square and square root.

A full multivariable model was fitted that included all candidate predictors with chosen transformations. The number of predictors was reduced with backward stepwise selection. We applied Akaike's information criterion [17] for predictor selection, which corresponds to a $p$ value of 0.157 for a predictor with one regression coefficient. Interaction terms between predictors were assessed, but not included because their predictive effects were very limited. The regression coefficients in the final model were multiplied with a shrinkage factor, which was estimated with bootstrapping $[9,18]$. Shrinkage is applied to obtain accurate predictions for new patients; without shrinkage, predictions are in general too extreme, resulting in low predictions being too low and high predictions too high.

The described analytical strategy to develop the prediction rule is in accordance with the current state of the art [9]. The strategy aims to develop a prediction rule that can provide accurate predictions for future patients rather than predictions that are correct for patients of the development dataset. Therefore, the selection of predictors in the model is based on multivariable modelling only with a backward selection procedure and liberal $p$ value ( 0.157 in this study). Use of higher $p$ values results in inclusion of relatively weaker predictors in the model at the cost of possible selection of a nuisance variable. Such a model performs well in new patients [19]. Further, the backward selection procedure starts with a limited number of candidate predictors with continuous variables studied in their original form (i.e. continuously). Dichotomising or categorising continuous variables may be seen as introducing an extreme form of rounding, with an inevitable loss of information [20].

The final model with the shrunken regression coefficients was presented as a score chart to facilitate clinical application. Scores were calculated by dividing the products of regression coefficients and predictor values by the smallest product. A constant was subtracted or added to rescale the scores in positive integers. The sum scores were then related to predicted risks.

The performance of the prediction rule was studied in terms of calibration (i.e. agreement between predicted risks and observed proportions of microalbuminuria) and discrimination. Discrimination was expressed with a concordance statistic ( $c$-statistic), which is similar to the area under the receiver-operating characteristic curve when dichotomous outcomes are considered [21]. The performance was studied in 100 bootstrap samples (internal validation) and in the 
independent validation sets of the EDC, FinnDiane and CACTI studies (external validation). Internal validation gives an impression of how much the performance of the prediction rule may deteriorate when applied to a new patient sample from the same population. External validation indicates how generalisable the prediction rule is to similar patients from other populations.

\section{Results}

Development of prediction rule The EURODIAB PCS cohort contained 1,115 patients with normal AER at baseline and normal AER or microalbuminuria at followup. Microalbuminuria was found in 143 (13\%) patients after a mean follow-up of 7.3 years. The mean age at baseline was 33 years, $49 \%$ of the patients were male and mean duration of diabetes was 14 years (Table 1).

Table 2 shows the univariable associations of the studied patient characteristics and progression to microalbuminuria. Continuous predictor values for this table were dichotomised at clinically relevant cut-offs or at the medians, in order to show interpretable odds ratios. Note that the multivariable analysis was performed with the continuous variables. Progression to microalbuminuria was associated with $\mathrm{HbA}_{1 \mathrm{c}}$, AER, all lipids, WHR, BMI and smoking.
The multivariable analysis indicated that $\mathrm{HbA}_{1 \mathrm{c}}, \mathrm{AER}$, WHR, BMI and smoking were the strongest predictors of progression to microalbuminuria (Table 3 ). The best transformation for $\mathrm{HbA}_{1 \mathrm{c}}$ was the natural logarithm (Fig. 1a). As a consequence, this variable was transformed into $\log _{e}\left(\mathrm{HbA}_{1 \mathrm{c}}\right.$-value). Linear associations with risk of microalbuminuria were observed for AER, WHR and BMI (Fig. 1b-d). The regression coefficients in the final model were multiplied with the estimated shrinkage factor of 0.88 (Table 3).

We distinguished four risk groups that were based on the sum scores (Table 4). The observed proportions and mean predicted risks were in good agreement across the four risk groups. The two highest risk groups together (sum scores $16-20$ and $\geq 21)$ contained $77 \%(n=83+27=110)$ of all 143 microalbuminuric patients of the EURODIAB PCS; the low-risk group (sum scores $2-10)$ contained only $1 \%(n=2)$ of microalbuminuric patients. The $c$-statistic was 0.71 , indicating reasonable discriminative ability. Internal validation reduced the $c$-statistic to 0.69 indicating minor optimism in the modelling procedure.

External validation of the prediction rule The EDC patients were slightly younger than patients of the development set (mean age 26 years compared with 33 years). The FinnDiane and CACTI patients were slightly older (36

Table 1 Characteristics of patients in the development and validation sets

\begin{tabular}{|c|c|c|c|c|}
\hline & EURODIAB PCS (1990-1991) & EDC (1986-1988) & FinnDiane (1998) & CACTI (2000-2002) \\
\hline Participants $(n)$ & 1,115 & 190 & 1,248 & 331 \\
\hline Age (years) & $33(9.6)$ & $26(8.1)$ & $36(12)$ & $37(9)$ \\
\hline Male sex, $n(\%)$ & $542(49)$ & $82(43)$ & $591(47)$ & $147(44)$ \\
\hline Diabetes duration (years) & $14(8.9)$ & $18(7.3)$ & $16(8.6)$ & $23(9)$ \\
\hline $\mathrm{HbA}_{1 \mathrm{c}}(\%)$ & $8.0(1.7)$ & $8.3(1.3)$ & $8.2(1.4)$ & $7.8(1.2)$ \\
\hline $\operatorname{AER}(\mu \mathrm{g} / \min )^{\mathrm{a}}$ & $8.2(0.91-20)$ & $8.5(0.83-20)$ & $5.2(0.30-20)$ & $5.0(0.5-20)$ \\
\hline Fasting triacylglycerol $(\mathrm{mmol} / \mathrm{l})^{\mathrm{a}}$ & $0.88(0.30-5.1)$ & $0.77(0.35-4.8)$ & $0.93(0.32-10)$ & $0.80(0.30-4.1)$ \\
\hline Non-HDL (mmol/l) & $3.6(1.0)$ & $3.1(0.72)$ & $3.5(0.91)$ & $3.0(0.80)$ \\
\hline $\mathrm{LDL}(\mathrm{mmol} / \mathrm{l})$ & $3.2(0.90)$ & $2.7(0.62)$ & $3.0(0.80)$ & $2.5(0.72)$ \\
\hline WHR, men & $0.88(0.07)$ & $0.86(0.04)$ & $0.89(0.07)$ & $0.86(0.06)$ \\
\hline WHR, women & $0.79(0.10)$ & $0.77(0.05)$ & $0.80(0.06)$ & $0.78(0.07)$ \\
\hline BMI, men $\left(\mathrm{kg} / \mathrm{m}^{2}\right)$ & $24(2.6)$ & $23(3.3)$ & $25(3.1)$ & $26(3.4)$ \\
\hline BMI, women $\left(\mathrm{kg} / \mathrm{m}^{2}\right)$ & $23(2.9)$ & $24(3.5)$ & $25(3.7)$ & $26(4.9)$ \\
\hline Diastolic pressure $(\mathrm{mmHg})$ & $73(11)$ & $68(8.7)$ & $78(9.0)$ & $77(8.4)$ \\
\hline Systolic pressure (mmHg) & $118(15)$ & $108(11)$ & $129(16)$ & $115(13)$ \\
\hline Pulse pressure (mmHg) & $44(12)$ & $40(9.0)$ & $51(13)$ & $39(11)$ \\
\hline Hypertension, $n(\%)$ & $148(13)$ & $2(1)$ & $176(14)$ & $107(32)$ \\
\hline Ever smoked, $n(\%)$ & $498(45)$ & $52(27)$ & $507(41)$ & $65(20)$ \\
\hline \multicolumn{5}{|l|}{ Outcome at follow-up } \\
\hline Microalbuminuria, $n(\%)$ & $143(13)$ & $34(18)$ & $96(7.7)$ & $20(6.0)$ \\
\hline
\end{tabular}

Values are the mean (SD) unless otherwise noted

${ }^{a}$ Median (range) 
Table 2 Univariable associations of patient characteristics assessed at baseline with progression to microalbuminuria

\section{Patient characteristic}

and

\section{Age}

$<30$ years

$\geq 30$ years

Sex

Men

Women

Duration

$<15$ years

$\geq 15$ years

$\mathrm{HbA}_{1 \mathrm{c}}$

$<8.0 \%$

$\geq 8.0 \%$

AER

$<8 \mu \mathrm{g} / \mathrm{min}$

$\geq 8 \mu \mathrm{g} / \mathrm{min}$

Fasting triacylglycerol

$<1.0 \mathrm{mmol} / \mathrm{l}$

$\geq 1.0 \mathrm{mmol} / 1$

Non-HDL

$<3.5 \mathrm{mmol} / 1$

$\geq 3.5 \mathrm{mmol} / 1$

LDL

$<3.0 \mathrm{mmol} / 1$

$\geq 3.0 \mathrm{mmol} / 1$

WHR

Men

$<0.95$

$\geq 0.95$

Women

$<0.85$

$\geq 0.85$

BMI

Men

$$
<25 \mathrm{~kg} / \mathrm{m}^{2}
$$$$
\geq 25 \mathrm{~kg} / \mathrm{m}^{2}
$$

Women

$$
\begin{array}{ll}
<25 \mathrm{~kg} / \mathrm{m}^{2} \\
\geq 25 \mathrm{~kg} / \mathrm{m}^{2}
\end{array}
$$

Pulse pressure

$<40 \mathrm{mmHg}$

$\geq 40 \mathrm{mmHg}$

Hypertension

No

Yes

Smoking

Never

Ever

Total

$\frac{\text { Normo-albuminuria }}{n}$

Micro-albuminuria

OR

$95 \%$ CI

$n \quad \%$

$\%$$$
435
$$$$
537
$$

87

63

13

87

80

13

1.0

$0.72-1.5$

469

86

73

70

14

12

0.89

$0.63-1.3$

610

87

91

13

13

1.0

$0.67-1.4$

558

414

92

52

\section{8}

82

52

91

18

2.4

$1.6-3.4$

491

481

91

46

97

9

83

17

2.2

$1.5-3.1$

643

329

90

75

10

83

68

17

1.8

$1.2-2.5$

498

474

90

56

10

84

87

16

1.6

1.1-2.3

449

90

50

10

85

93

15

1.6

1.1-2.3

417

87

82

62

11

13

18

1.4

0.70-2.9

425

78

89

54

16

11

17

1.6

0.88-3.0

83

347

122

89

43

11

80

30

20

2.0

$1.2-3.3$

389

114

89

49

11

84

21

16

1.5

$0.84-2.5$

368

86

58

14

604

88

85

12

0.89

$0.62-1.3$

844

87

123

20

13

86

14

1.1

$0.65-1.8$

550

89

67

11

$\begin{array}{lll}85 & 76 & 15\end{array}$

87

15

1.5

$1.0-2.1$ 
Table 3 Multivariable associations of selected predictors for progression to microalbuminuria

\begin{tabular}{|c|c|c|c|}
\hline Predictor & OR & $95 \% \mathrm{CI}$ & $\beta$ coefficient $^{\circ}$ \\
\hline $\mathrm{HbA}_{1 \mathrm{c}}(\%)$, transformed $^{\mathrm{b}}$ & 1.26 & $1.09,1.45$ & 0.206 \\
\hline $\operatorname{AER}(\mu \mathrm{g} / \mathrm{min})$ & 1.09 & $1.07,1.11$ & 0.076 \\
\hline WHR, transformed ${ }^{\mathrm{c}}$ & 1.20 & $0.90,1.61$ & 0.163 \\
\hline $\operatorname{BMI}\left(\mathrm{kg} / \mathrm{m}^{2}\right)$ & 1.07 & $1.03,1.10$ & 0.059 \\
\hline Ever smoking & 1.40 & $1.16,1.69$ & 0.300 \\
\hline Intercept & & & -9.883 \\
\hline$c$-statistic ${ }^{\mathrm{d}}$ & & 0.71 & 0.69 \\
\hline
\end{tabular}

${ }^{\mathrm{a}} \beta$ coefficients were multiplied by a shrinkage factor of 0.88 to improve predictions in future patients

${ }^{\mathrm{b}} \log _{e}\left(\mathrm{HbA}_{1 \mathrm{c}}\right) \times 10$

${ }^{\mathrm{c}} \mathrm{WHR} \times 10$

${ }^{\mathrm{d}}$ Estimated in the development set and corrected for optimism after bootstrapping

and 37). Occurrence of hypertension varied between $1 \%$ (EDC) and $32 \%$ for the validation sets, with $13 \%$ in the development set. Progression to microalbuminuria occurred in $18 \%$ (EDC), $8 \%$ (FinnDiane) and $6 \%$ (CACTI) of patients (Table 1).

Most of the microalbuminuric patients were categorised in one of the two groups with the highest risk: $27 / 34(79 \%)$ for EDC, 81/96 (84\%) for FinnDiane and 16/20 (80\%) for CACTI (Table 4), which is similar to the results found in the development set. The discriminative ability of the prediction rule was similar to that in the development set for the EDC patients with $c$-statistic $=0.72$. The discriminative ability was even better in the FinnDiane and CACTI patients $(c$-statistic $=0.79$ in both).

The mean predicted risks in the two datasets with recently treated patients (FinnDiane and CACTI) were higher than the observed proportions of microalbuminuria (13\% predicted risk and $8 \%$ observed in FinnDiane, $11 \%$ and $6 \%$ in CACTI). We adjusted the intercept of the prediction rule in such a way that the mean predicted risk agrees with the observed proportion of microalbuminuria (intercept changes from -9.88 to -10.14 ).

Figure 2 shows a score chart that corresponds to the adjusted prediction rule. The score chart facilitates calculation of the predicted risk of microalbuminuria for an individual patient who is currently treated. Values for continuous predictors are given in small steps. An individual sum score consists of the sum of all scores. The lower part of the figure shows the predicted risks that correspond to the sum scores.

\section{Discussion}

We developed and validated a clinical prediction rule that can be used to predict progression from normal AER to microalbuminuria in type 1 diabetes patients. First, we identified a series of predictors for the risk of progression to microalbuminuria within a period of 7 years. The following five characteristics predominantly contributed to prediction in a multivariable logistic regression model: $\mathrm{HbA}_{1 \mathrm{c}}, \mathrm{AER}$, WHR, BMI and ever smoking. These predictors can be relatively easily obtained by physical examination and laboratory tests. External validation of the prediction rule in three independent patient samples showed good performance.

$\mathrm{HbA}_{1 \mathrm{c}}$ and baseline AER have consistently been shown to be important predictors of microalbuminuria $[2-5,22]$ and were also included in a previous model developed by Rossing and co-workers [5]. Smoking was also included in both models. In contrast, BMI and WHR were included in the present model, but were not considered in the development of the Rossing model, which did, however, include retinopathy. We found with the EURODIAB data that the present model discriminated patients with microalbuminuria from those with normal AER better than the Rossing model, which included retinopathy (c-statistics 0.69 and 0.65 respectively). A model that included the four predictors of the Rossing model $\left(\mathrm{HbA}_{1 \mathrm{c}}, \mathrm{AER}\right.$, current smoking and any retinopathy) fitted with the EURODIAB PCS data showed a $c$-statistic of 0.66 . The additive predictive value for WHR in a model that already includes BMI indicates that central obesity contains different information than general obesity. This is confirmed by the
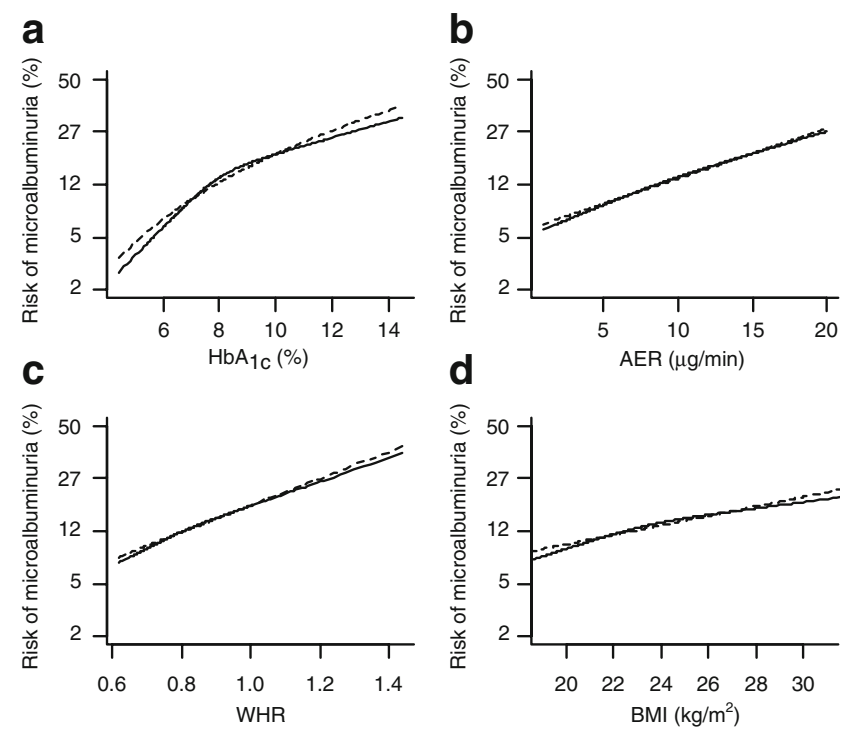

Fig. 1 Nature of the univariable associations of the continuous predictors a $\mathrm{HbA}_{1 \mathrm{c}}$, b AER, $\mathbf{c}$ WHR and $\mathbf{d} \mathrm{BMI}$ with the risk of microalbuminuria. Continuous lines, restricted cubic splines with three knots; dotted lines, the chosen transformations. Transformations: a $\log _{e}$, b-d linear. The deviant scaling on $y$-axes corresponds to the logodds or logit of microalbuminuria, which is the adequate scale to study the shape of the association 
Table 4 Observed proportions and predicted risks of microalbuminuria among four risk groups in the development set (EURODIAB PCS) and validation sets (EDC, FinnDiane and CACTI)

\begin{tabular}{|c|c|c|c|c|c|c|c|c|c|c|c|c|}
\hline \multirow[t]{3}{*}{ Sum score } & \multicolumn{3}{|c|}{ EURODIAB PCS } & \multicolumn{3}{|l|}{ EDC } & \multicolumn{3}{|c|}{ FinnDiane } & \multicolumn{3}{|l|}{ CACTI } \\
\hline & \multicolumn{2}{|c|}{$\begin{array}{l}\text { Observed } \\
\text { proportion }\end{array}$} & \multirow{2}{*}{$\begin{array}{l}\text { Mean } \\
\text { predicted } \\
\text { risk } \\
\%\end{array}$} & \multicolumn{2}{|c|}{$\begin{array}{l}\text { Observed } \\
\text { proportion }\end{array}$} & \multirow{2}{*}{$\begin{array}{l}\text { Mean } \\
\text { predicted } \\
\text { risk } \\
\%\end{array}$} & \multicolumn{2}{|c|}{$\begin{array}{l}\text { Observed } \\
\text { proportion }\end{array}$} & \multirow{2}{*}{$\begin{array}{l}\text { Mean } \\
\text { predicted } \\
\text { risk } \\
\%\end{array}$} & \multicolumn{2}{|c|}{$\begin{array}{l}\text { Observed } \\
\text { proportion }\end{array}$} & \multirow{2}{*}{$\begin{array}{l}\text { Mean } \\
\text { predicted } \\
\text { risk } \\
\%\end{array}$} \\
\hline & $n / N$ & $\%$ & & $n / N$ & $\%$ & & $n / N$ & $\%$ & & $n / N$ & $\%$ & \\
\hline $2-10$ & $2 / 73$ & 3 & 3 & $0 / 5$ & 0 & 4 & $1 / 61$ & 2 & 4 & $1 / 22$ & 5 & 4 \\
\hline $11-15$ & $31 / 470$ & 7 & 8 & $7 / 88$ & 8 & 8 & $14 / 564$ & 3 & 8 & $3 / 196$ & 2 & 8 \\
\hline $16-20$ & $83 / 485$ & 17 & 16 & $22 / 83$ & 27 & 15 & $47 / 509$ & 9 & 15 & $12 / 93$ & 13 & 15 \\
\hline$\geq 21$ & $27 / 87$ & 31 & 32 & $5 / 14$ & 36 & 31 & $34 / 114$ & 30 & 32 & $4 / 20$ & 20 & 31 \\
\hline
\end{tabular}

relatively low correlation between WHR and BMI (Pearson correlation $r=0.21$ ). The differences in effect of central and general obesity have also been described for the risk of cardiovascular events [23].

The predictors hyperglycaemia and central obesity are part of the insulin resistance syndrome [24]. Insulin resistance is also a risk factor for the development of diabetic complications. We further studied the added value of insulin sensitivity with the amount of fasting insulin (per $\mathrm{kg}$ body weight). However, this factor did not have added value in our model $(p=0.53)$.

Continuous predictors are best included in a model as such and not categorised [20]. In this way, all information is used for the prediction. Continuous variables do not necessarily have to be included as linear terms. We found that logarithmic transformation of $\mathrm{HbA}_{1 \mathrm{c}}$ predicts the risk of microalbuminuria better than the frequently used linear values. The nature of the relation between AER and risk of microalbuminuria was linear, although a logarithmic transformation of AER is frequently used [3, 5]. Apparently, a logarithmic transformation of AER is only necessary to estimate correct values of the mean and standard deviation, given the skewed distribution of AER.

We studied the generalisability of our prediction rule with respect to place (EDC), time (FinnDiane) and time and place (CACTI). The US cohort (EDC) with patients treated in the same time period as the patients from the development set (the 1990s) showed similar discriminative ability of the prediction rule $(c$-statistic $=0.71$ compared with 0.69 in the development set). $C$-statistic values around 0.7 indicate reasonable discriminative ability for prognostic models. Prediction of an event later in time (here 7 years) is more difficult than prediction of an event shortly after the baseline measurement. Furthermore, the predicted risks were reasonably in agreement with the observed proportions of microalbuminuria (Table 4).

It was particularly important to study the generalisability of the prediction rule in time, since treatment of type 1 diabetes patients with normal AER has changed. Nowadays, ACE inhibitors and statins are prescribed more frequently to lower blood pressure and lipid plasma concentrations respectively. The use of these medications has been shown to affect the transition from normo- to microalbuminuria in type 1 diabetes patients $[25,26]$, as was also apparent from our data. The incidence of microalbuminuria was only $7.7 \%$ in FinnDiane and $6.0 \%$ in CACTI compared with $13 \%$ in the earlier development sample. Indeed, predicted risks were too high for the patients from the FinnDiane and CACTI studies. However, the model was well able to discriminate

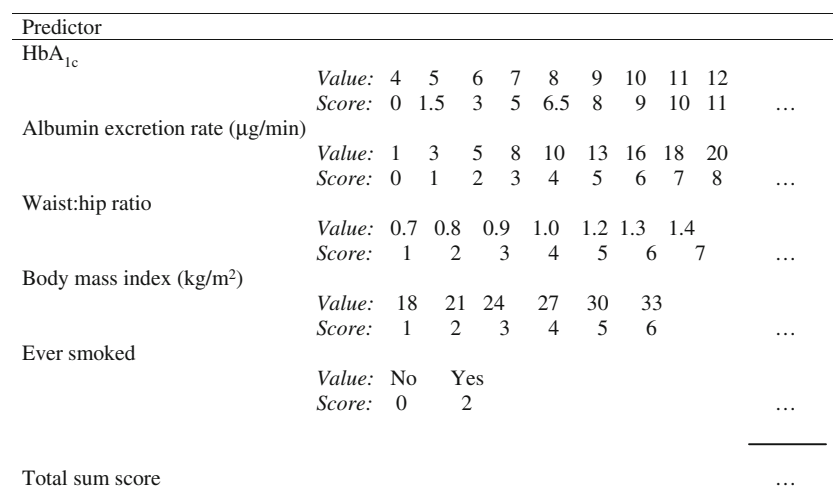

\begin{tabular}{|lccccccccccccc|}
\hline Total sumscore & 6 & 8 & 10 & 12 & 14 & 16 & 18 & 20 & 22 & 24 & 26 & 28 & 30 \\
\hline $\begin{array}{l}\text { Risk of micro- } \\
\text { albuminuria (\%) }\end{array}$ & 1.5 & 2 & 3 & 4.5 & 6 & 9 & 14 & 19 & 25 & 32 & 43 & 54 & 63 \\
\hline
\end{tabular}

Fig. 2 Score chart to predict risk of microalbuminuria in patients with type 1 diabetes. The scores are derived from the prediction rule that contain the shrunken regression coefficients of the final model and updated intercept: $1 \mathrm{p}=-10.14+0.21 \times \log _{e}\left(\mathrm{HbA}_{1 \mathrm{c}}\right)+0.08 \times$ $\mathrm{AER}+1.63 \times \mathrm{WHR}+0.06 \times \mathrm{BMI}+0.30 \times$ ever-smoking, where $\log _{e}\left(\mathrm{HbA}_{1 \mathrm{c}}\right)$ is the natural logarithm of $\mathrm{HbA}_{1 \mathrm{c}}$, AER is expressed in $\mu \mathrm{g} / \mathrm{min}, \mathrm{BMI}$ is expressed in $\mathrm{kg} / \mathrm{m}^{2}$ and ever-smoking is set at 1 if true and 0 if false. Risk of microalbuminuria $=1 /(1+\exp [-\operatorname{lp}])$. A hypothetical patient with $\mathrm{HbA}_{1 \mathrm{c}}$ of $6 \%$ (3 points), AER of $5 \mu \mathrm{g} / \mathrm{min}$ ( 2 points), WHR of 0.9 (3 points), BMI of 24 (3 points) and who has never smoked ( 0 points) has a sum score of 11 , which corresponds to a risk of $4 \%$ 
between patients with and without microalbuminuria. Therefore, a simple recalibration step was sufficient to make the rule valid for recently treated patients.

The clinical implications of our study are that risk of microalbuminuria can be established at an early stage and patient management tailored to risk levels. We categorised the patients into four risk groups with scores 2 to 10,11 to 15,16 to 20 , and 21 and higher. The highest two risk groups together contained $77 \%(110 / 143)$ of all microalbuminuric patients of the development set and 79\%,80\% and $84 \%$ of all microalbuminuric patients of the validation sets. We would advocate offering these high-risk patients more frequent check-ups than once a year, perhaps together with the quarterly routine visit and with an overnight or $24 \mathrm{~h}$ urine collection to measure the albumin:creatinine ratio. Current surveillance protocols recommend follow-up of patients more frequently than once a year only after an abnormal surveillance result. Under this protocol, a considerable proportion of new microalbuminuria cases will be recognised later than necessary. The identification, by our prediction rule, of a group of patients with normal AER and at high risk of progressing to microalbuminuria might facilitate the early introduction of ACE inhibitor or angiotensin receptor blocker therapy, if trials currently under way suggest benefit. Glycaemic control could also be intensified and other risk factors for microalbuminuria, e.g. smoking, BMI and WHR could be more strictly controlled than is usual. It is currently unknown whether such intervention strategies based on our prediction rule would be of benefit; this should be the focus of future research.

Unfortunately, the model predicts for one occasion only, i.e. after 7 years of follow-up. The assessment of the outcome only after 7 years has two implications. First, we do not have information on time of onset of microalbuminuria. Time-toevent analysis, e.g. with Cox proportional hazards regression analysis, was therefore not possible. Second, we do not know how many patients remain microalbuminuric and how many regress. Several studies have shown regression of microalbuminuria in type 1 diabetes patients [2, 27, 28]. Cumulative incidences of regression vary between $13 \%$ and $56 \%$ and are mainly induced by intensive therapy. Only few patients with microalbuminuria undergo spontaneous regression that is permanent (around 15\%) [2]. Consequently, our rule may only slightly overestimate a patient's risk of progression to microalbuminuria.

In conclusion, we developed a prediction rule to estimate the risk of progressing to microalbuminuria in individual type 1 diabetes patients. The rule was developed in a European cohort and externally validated in two US cohorts and another European cohort. We believe that this prediction rule could be used to divide patients into different risk categories. Such risk categories could guide surveillance recommendations and ultimately improve the prevention of long-term chronic complications.

Acknowledgements We would like to thank all study participants who volunteered their time, all the staff involved in the EURODIAB PCS, EDC, FinnDiane and CACTI studies, and all investigators and consultants. EURODIAB PCS was financially supported by the Wellcome Trust, the European Community and Diabetes UK. The EDC study was supported by National Institutes of Health Grant R01 DK34818. The FinnDiane Study was supported by the Folkhälsan Research Foundation, Wilhelm and Else Stockmann Foundation, Liv och Hälsa Foundation, Signe and Ane Gyllenberg Foundation, Sigrid Juselius Foundation and the European Commission (QLG2-CT2001-01669; LSHB-CT-2003-503364 and LSHB-CT-2006-037681). Support for the CACTI study was provided by the National Institutes of Health grants R01 HL61753 and R01 HL079611, American Diabetes Association grant 7-09-CVD-06 and Diabetes Endocrinology Research Center Clinical Investigation Core P30 DK57516. The study was performed at the Adult General Clinical Research Center at the University of Colorado Denver Anschutz Medical Center (supported by NIH grant M01 RR000051) and at the Barbara Davis Center for Childhood Diabetes. Y. Vergouwe and K. G. M. Moons were supported by the Netherlands Organization for Scientific Research Grant ZON-MW 917.46.360.

Duality of interest The authors declare that there is no duality of interest associated with this manuscript.

Open Access This article is distributed under the terms of the Creative Commons Attribution Noncommercial License which permits any noncommercial use, distribution, and reproduction in any medium, provided the original author(s) and source are credited.

\section{References}

1. Orchard TJ, Dorman JS, Maser RE et al (1990) Prevalence of complications in IDDM by sex and duration. Pittsburgh Epidemiology of Diabetes Complications Study II. Diabetes 39:1116-1124

2. Hovind P, Tarnow L, Rossing P et al (2004) Predictors for the development of microalbuminuria and macroalbuminuria in patients with type 1 diabetes: inception cohort study. BMJ 328:1105

3. Coonrod BA, Ellis D, Becker DJ et al (1993) Predictors of microalbuminuria in individuals with IDDM. Pittsburgh Epidemiology of Diabetes Complications Study. Diabetes care 16:1376-1383

4. Group MCS (1999) Predictors of the development of microalbuminuria in patients with type 1 diabetes mellitus: a seven-year prospective study. Diabet Med 16:918-925

5. Rossing P, Hougaard P, Parving HH (2002) Risk factors for development of incipient and overt diabetic nephropathy in type 1 diabetic patients: a 10-year prospective observational study. Diabetes care 25:859-864

6. Mogensen CE, Christensen CK (1984) Predicting diabetic nephropathy in insulin-dependent patients. N Engl J Med 311:89-93

7. Almdal T, Norgaard K, Feldt-Rasmussen B, Deckert T (1994) The predictive value of microalbuminuria in IDDM. A five-year followup study. Diabetes care 17:120-125

8. Krolewski AS, Kosinski EJ, Warram JH et al (1987) Magnitude and determinants of coronary artery disease in juvenile-onset, insulin-dependent diabetes mellitus. Am J Cardiol 59:750-755

9. Harrell FE Jr, Lee KL, Mark DB (1996) Multivariable prognostic models: issues in developing models, evaluating assumptions and 
adequacy, and measuring and reducing errors. Stat Med 15:361387

10. Steyerberg EW, Eijkemans MJC, Harrell FE Jr, Habbema JDF (2000) Prognostic modelling with logistic regression analysis: a comparison of selection and estimation methods in small data sets. Stat Med 19:1059-1079

11. Group EICS (1994) Microvascular and acute complications in IDDM patients. Diabetologia 37:278-285

12. Thorn LM, Forsblom C, Fagerudd J et al (2005) Metabolic syndrome in type 1 diabetes: association with diabetic nephropathy and glycemic control (the FinnDiane study). Diabetes Care 28:2019-2024

13. Dabelea D, Kinney G, Snell-Bergeon JK et al (2003) Effect of type 1 diabetes on the gender difference in coronary artery calcification: a role for insulin resistance? The Coronary Artery Calcification in Type 1 Diabetes (CACTI) Study. Diabetes 52: 2833-2839

14. Kearney EM, Mount JN, Watts GF, Slavin BM, Kind PR (1987) Simple immunoturbidimetric method for determining urinary albumin at low concentrations using Cobas-Bio centrifugal analyser. J Clin Pathol 40:465-468

15. Ellis D, Buffone GJ (1977) New approach to evaluation of proteinuric states. Clin Chem 23:666-670

16. Chaturvedi N, Sjoelie AK, Porta M et al (2001) Markers of insulin resistance are strong risk factors for retinopathy incidence in type 1 diabetes. Diabetes Care 24:284-289

17. Atkinson AC (1980) A note on the generalized information criterion for choice of a model. Biometrika 67:413-418

18. Efron B, Tibshirani RJ (1993) An introduction to the bootstrap. Chapman and Hall, London
19. Steyerberg EW, Eijkemans MJ, Habbema JD (1999) Stepwise selection in small data sets: a simulation study of bias in logistic regression analysis. J Clin Epidemiol 52:935-942

20. Royston P, Altman DG, Sauerbrei W (2006) Dichotomizing continuous predictors in multiple regression: a bad idea. Stat Med 25:127-141

21. Harrell FE Jr, Califf RM, Pryor DB, Lee KL, Rosati RA (1982) Evaluating the yield of medical tests. JAMA 247:2543-2546

22. Chaturvedi N, Bandinelli S, Mangili R, Penno G, Rottiers RE, Fuller JH (2001) Microalbuminuria in type 1 diabetes: rates, risk factors and glycemic threshold. Kidney Int 60:219-227

23. Kannel WB, Cupples LA, Ramaswami R, Stokes J 3rd, Kreger BE, Higgins M (1991) Regional obesity and risk of cardiovascular disease; the Framingham Study. J Clin Epidemiol 44:183-190

24. Williams KV, Erbey JR, Becker D, Arslanian S, Orchard TJ (2000) Can clinical factors estimate insulin resistance in type 1 diabetes? Diabetes 49:626-632

25. Group TES (1997) Randomised placebo-controlled trial of lisinopril in normotensive patients with insulin-dependent diabetes and normoalbuminuria or microalbuminuria. Lancet 349:1787-1792

26. Douglas K, O'Malley PG, Jackson JL (2006) Meta-analysis: the effect of statins on albuminuria. Ann Intern Med 145:117-124

27. Giorgino F, Laviola L, Cavallo Perin P, Solnica B, Fuller J, Chaturvedi N (2004) Factors associated with progression to macroalbuminuria in microalbuminuric type 1 diabetic patients: the EURODIAB Prospective Complications Study. Diabetologia 47:1020-1028

28. Perkins BA, Ficociello LH, Silva KH, Finkelstein DM, Warram JH, Krolewski AS (2003) Regression of microalbuminuria in type 1 diabetes. N Engl J Med 348:2285-2293 